\title{
Multiple birthdating analyses in adult neurogenesis: a line-up of the usual suspects
}

\section{María Llorens-Martín and José L. Trejo*}

Department of Molecular, Cellular and Developmental Neurobiology, Cajal Institute, Madrid, Spain

\section{Edited by:}

Silvia De marchis, University of Turin,

Italy

\section{Reviewed by:}

Muriel Koehl, Institut national de la santé et de la recherche médicale

(Inserm), France

Helena Mira, Instituto de Salud Carlos III, Spain

\section{${ }^{*}$ Correspondence:}

José L. Trejo, Department of Molecular, Cellular and Developmental

Neurobiology, Cajal Institute, Av.

Doctor Arce, 37, 28002 Madrid, Spain.

e-mail:jltrejo@cajal.csic.es
Analyzing the variation in different subpopulations of newborn neurons is central to the study of adult hippocampal neurogenesis. The acclaimed working hypothesis that different subpopulations of newborn, differentiating neurons could be playing different roles arouses great interest. Therefore, the physiological and quantitative analysis of neuronal subpopulations at different ages is critical to studies of neurogenesis. Such approaches allow cells of different ages to be identified by labeling them according to their probable date of birth. Until very recently, only neurons born at one specific time point could be identified in each experimental animal. However the introduction of different immunohistochemically compatible markers now enables multiple subpopulations of newborn neurons to be analyzed in the same animal as in a line-up, revealing the relationships between these subpopulations in response to specific influences or conditions. This review summarizes the current research carried out using these techniques and outlines some of the key applications.

Keywords: neurogenesis, dentate gyrus, dual birthdating, CldU, IdU, BrdU

\section{INTRODUCTION}

The accurate labeling of newborn cells in the adult brain poses a fundamental challenge in the study of adult neurogenesis. Adult brain neurogenesis is closely linked with learning and memory, and it has also been implicated in anxiety and depression (for recent reviews, see Aimone et al., 2010; Deng et al., 2010). Furthermore, many parameters associated with adult neurogenesis are altered in models of Alzheimer's and other neurodegenerative diseases, and in models of ischemia or schizophrenia. Adult neurogenesis has attracted considerable attention due to the cellular properties observed during this period, which may be adapted to rescue neuronal loss due to aging or neurodegenerative processes. In the adult hippocampus, newborn neurons are granule neurons of the dentate gyrus, whose precursors reside locally in the inner side of the granular layer, known as the subgranular zone (SGZ). The cells born in the adult dentate gyrus are derived from neural stem cells (NSC; type-1 cells); these cells are either quiescent (a small proportion) or divide slowly, to generate another group of rapidly dividing cells known as intermediate progenitor cells (type-2a cells). The progeny of these cells are neuroblasts that either continue to proliferate or exit the cell cycle to mature and differentiate into granule neurons, apparently indistinguishable from the rest of the dentate gyrus granule neurons. It will be important to note here that these cell populations can be traced using relatively specific markers (Figure 1). These markers can be easily used in conjunction with "birth-marking" labels to determine that the different subpopulations were actually born in the adult brain.

In recent years, the labeling of newly born cells in the adult brain has been almost overwhelmingly ruled by the use of 5-bromo$3^{\prime}$-deoxy-uridine (BrdU). "The underlying principle is straightforward: a permanent marker is brought into a cell of interest at the time point of division and the later fate of this cell is studied. Because the marker is persistent, it is possible to retrospectively conclude with confidence that a marked cell must have undergone cell division at the time when the marker was injected (sic)" (Kempermann, 2006). The protocol involves the administration of the thymidine analog (normally by intraperitoneal injection, and to a lesser extent via drinking water) which incorporates into the dual helix of any cell actively synthesizing DNA during the period the product remains systemically available and active in the body (usually around $2 \mathrm{~h}$ or less). After the animal is sacrificed, staining is detected by immunohistochemistry using antibodies specifically directed against the analog.

BrdU has been the marker of choice in recent years for several reasons, in part because this method requires no radioactivity unlike the use of tritiated thymidine, which for decades was used to label dividing cell populations during brain development. Furthermore, BrdU staining is readily detected by immunohistochemistry. The only significant drawback of this technique is the requirement to unmask the epitopes recognized by the primary antibody against BrdU using hydrochloric acid. Naturally there some other minor issues are involved in its use, though these have largely been solved over the years.

One of the most interesting parameters that can be assessed by the labeling of dividing cells is survival time, defined as the time between the incorporation of the BrdU and that of the animal's sacrifice. The age of labeled cells is equivalent to the survival time (i.e., cells are 1 month old if the animal injected with BrdU was sacrificed 1 month after BrdU injection).

A key limitation of this method is its ability to recognize only a single pool of BrdU incorporated into the body, regardless of when and how it was administered. This has significant implications: all incorporated BrdU is detected as a single signal, and hence, the cell populations that have incorporated BrdU are indistinguishable. Therefore, BrdU administration must be sufficiently discrete in time (depending on the experimental design) 


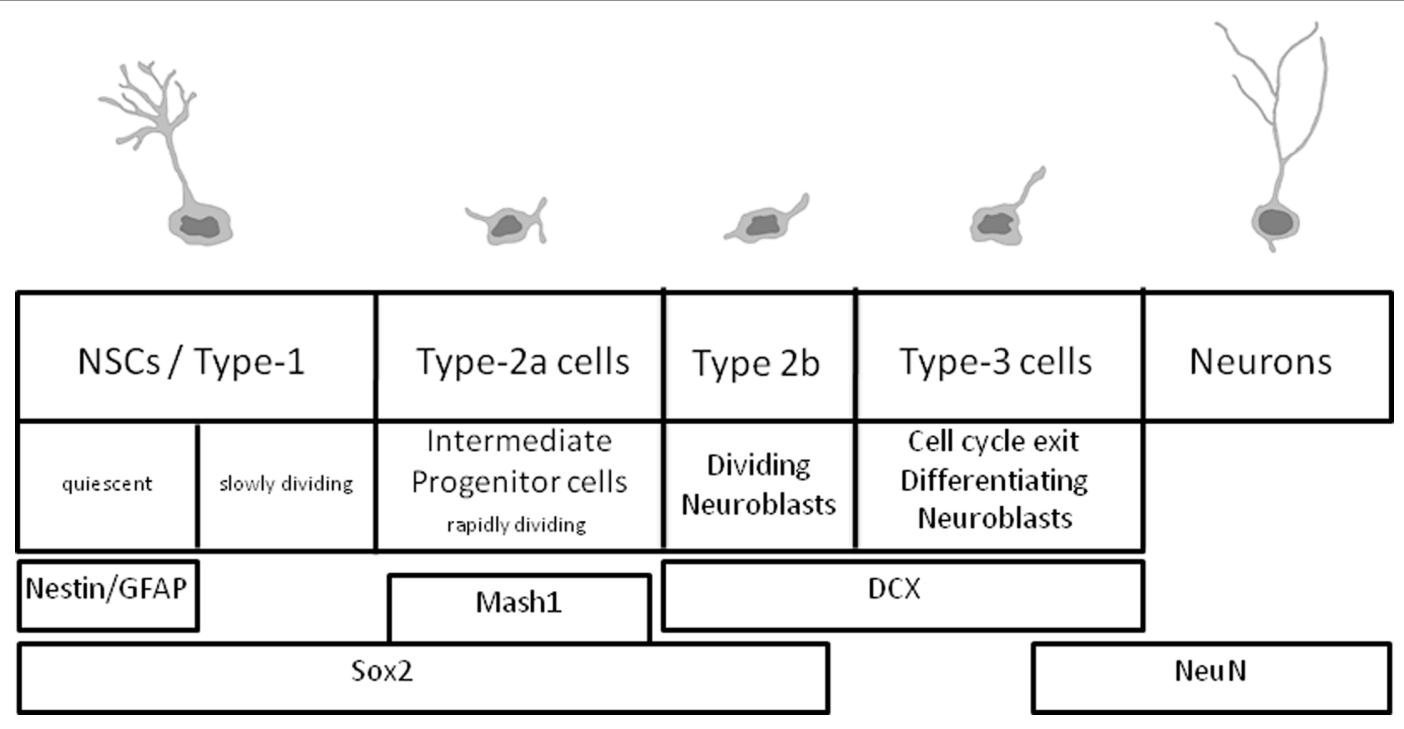

FIGURE 1 | Summary of the cell types in adult hippocampal neurogenesis and the expression of the main markers.

so that cell populations to be marked by BrdU are also consistent in terms of age. This issue is particularly important in short experiments (i.e., days). If the animal is injected with BrdU over $n$ days, and sacrificed 1 week after the last injection, the cohort of labeled cells is considered to be between 7 and $7-n$ days old. Therefore, the injection rate in conjunction with the survival time can produce large differences, and in the worst cases, cell populations that are markedly heterogeneous in terms of age may be labeled equally. Within the framework of the experimental design, the investigator must judge what heterogeneity in terms of age can be accepted in the target cell population. In contrast, in a long-term survival experiment, several injections administered over consecutive days can label a cohort of cells that are subsequently identified as a single group, not taking into account the age difference between the cells marked by the first and the last injection.

This inherent limitation of BrdU labeling implies that each animal can only contain a population of cells marked, as homogeneous as possible in terms of age, precluding any distinction between cells of very different ages. BrdU injections at two times sufficiently separated would generate similarly labeled cells although they are very different. For this reason, the data from cell populations with qualitatively different ages has been achieved by using different experimental groups (different animals) that were subjected to different injection regimes of BrdU and/or different survival times (e.g., group A is sacrificed after 1 week to analyze 1 week old cells, while group B is sacrificed after 1 month to analyze 1 month old cells). This approach has generated a large amount of data. However, it is not a trivial matter that these comparisons between cell populations of different age, born in the adult brain, have been analyzed to date in different animals. It is clear that the study of possible direct relationships between subpopulations of qualitatively different age is strengthened considerably when performed in the same animal, avoiding potential confounding factors due to any intra-group variability between individuals. This problem can now be avoided by the use of a recent technique that permits two or three cell subpopulations of different ages to be labeled in the same animal.

This method involves the injection of the thymidine analogs 5-iodo-2'-deoxy-uridine (IdU) and 5-chloro-2'-deoxy-uridine (CldU), which can be unequivocally distinguished from one other using respective antibodies anti-CldU and anti-IdU, labeling two populations of different ages in the same animal.

It only takes a dual immunohistochemistry to detect the two cell populations of different age born in the adult brain, triple immunohistochemistry in the event that is also to characterize the phenotype of these two subpopulations.

The first attempts to cope with the problem were not so simple. Certainly, the first works reporting the separate detection of two different halogenated nucleotides that have been incorporated in DNA, used immunofluorescence dual-staining methods in vitro (Shibui et al., 1989; Bakker et al., 1991; Aten et al., 1992). However, these methods required complex histological procedures to distinguish between the two antibodies.

By using this technique, an early study by Manders et al. (1992) compared different replication patterns by using immunofluorescence dual staining of cell nuclei in vitro, after incorporating two independent markers of DNA replication in the same nucleus, and recording fluorescence signals with a dual-color confocal microscope. However, one of the first studies reporting the use of dual birthdate labeling in the brain used BrdU and IdU in human patients (Hoshino et al., 1992). The authors injected brain tumors patients with BrdU, followed $5 \mathrm{~h}$ later by IdU. The percentage of $\mathrm{BrdU}^{+}$cells was used to establish the $\mathrm{S}$ phase fraction, while the IdU/ $\mathrm{BrdU}$ and BrdU/IdU ratios were used to establish the duration of the S phase and other parameters of cell cycle kinetics. Subsequently, Burns and Kuan (2005) were able to distinguish different cell populations depending on the embryonic day the cells were generated during cortical development, by using antibodies raised in different species (rat and mouse) to distinguish two deoxyuridines 
(again BrdU and IdU), and further incubation with species-specific secondary antibodies conjugated to different fluorophores. This method permits the separate evaluation of dual-labeled cells (BrdU and IdU signal) and single-labeled cells (BrdU signal). This way, the authors also demonstrated the length of the $S$ phase of neural progenitor cells in the adult mouse dentate gyrus.

\section{CHARACTERIZATION AND SET-UP}

A number of papers have described different approaches to implement these protocols. In most of them, both thymidine analogs were injected in the same animal to a number of individuals. The first consideration must be the analog dose injected. For this purpose, three important factors have to be taken into account in the use of two thymidine analogs at a time: (i) the absence of cross-reactivity in the immunohistochemistry, (ii) the possibility to detect them along with markers of lineage/differentiation, making possible the identification of the maturational state of the newborn cell/neuron and nevertheless, (iii) the ability to quantify the number of these cells by means of stereology. Each of these requirements can be fulfilled by the use of equimolar administration of CldU and IdU to the animals, as demonstrated by Vega and Peterson (2005).

For this goal, we have used in mice doses of CldU and IdU that are equimolar to the BrdU dose. Specifically, we use to prepare it according to the $50 \mathrm{mg} / \mathrm{kg}$ bw BrdU dose. This dose was selected because the cells with a nucleus completely filled by the labeling at this dose, or a nucleus containing easily identifiable patches of chromatin marked by the fluorophore at this dose, can clearly be identified as cells that were in S phase in time of administration of the thymidine analog, thus preventing to analyze cells with a weak incorporation possibly due to DNA repair or other unknown factors. The doses used were $42.75 \mathrm{mg} / \mathrm{kg}$ bw for CldU and $56.75 \mathrm{mg} /$ $\mathrm{kg}$ bw for IdU. The use of saturating doses of BrdU, CldU, and IdU has been described previously by Leuner et al. (2009).

Nevertheless, the doses of thymidine analogs used in the literature vary considerably. One of the most common approaches is to administer a first analog over a 2-3 week period, and after a variable intermediate period without labeling (see Re-entering the cell cycle), then the second analog over a subsequent 2-3 week period. This protocol has usually been achieved by administering the nucleosides in drinking water (see for example Maslov et al., 2004; Gobeske et al., 2009) at $1 \mathrm{mg} / \mathrm{ml}$ both nucleosides, while Bonaguidi et al. (2008) used $1.15 \mathrm{mg} / \mathrm{ml} \mathrm{CldU}$ and $0.85 \mathrm{mg} / \mathrm{ml}$ IdU with $2.5 \%$ sucrose. Other approaches consist of intraperitoneal injection of the analogs. This way the doses used has been heterogeneous either, see for example Bauer and Patterson (2005) using $16.67 \mathrm{mg} / \mathrm{ml} \mathrm{CldU}$ in saline and $10 \mathrm{mg} / \mathrm{ml} \mathrm{IdU}$ in $\mathrm{PBS} / \mathrm{NaOH}$, pH 8.0, while Gobeske et al. (2009), Mira et al. (2010), and Stone et al. (2010) used $10 \mathrm{mg} / \mathrm{ml} \mathrm{CldU}$ in saline and IdU equimolar to CldU, what means to use doses adjusted volumetrically to the molar equivalent of $50 \mathrm{mg} / \mathrm{kg}$ BrdU for each animal. The authors used $42.5 \mathrm{mg} / \mathrm{kg}$ CldU and $57.5 \mathrm{mg} / \mathrm{kg}$ IdU.

Although a number of authors used doses of CldU and IdU equimolar to that of BrdU, this BrdU dose itself varies in the literature from $50 \mathrm{mg} / \mathrm{kg}$ bw BrdU (as used by ourselves), some others used equimolar doses to $100 \mathrm{mg} / \mathrm{kg}$ bw (for example Breunig et al., 2007). A number of other authors have published the use of equimolar doses but not mentioning to what BrdU dose.
Despite the variability in the protocols used, and despite the completely different injection regimes used, all these studies report reliable and consistent labeling. In our studies, CldU can easily be prepared in $0.9 \%$ saline. IdU by using $0.1 \mathrm{M} \mathrm{PBS}$ with 2 drops of $5 \mathrm{~N} \mathrm{NaOH}$ added per 10-15 $\mathrm{ml}$ PBS. This solution was then heated 2-3 times in a microwave oven without boiling and vigorously stirred manually.

The next parameter to be taken into account is the survival time. Several groups have used different survival times in different groups of animals to characterize and set-up the technique. Specifically, we injected CldU and IdU at different time points in the same animals and they were then sacrificed either $24 \mathrm{~h}$ or 2 weeks after the last injection.

Finally, mice were anesthetized with pentobarbital, transcardially perfused with saline followed by $4 \%$ paraformaldehyde in phosphate buffer $(\mathrm{PB})$. The brains were removed, post-fixed overnight in the same fixative and sectioned with a vibratome $(50 \mu \mathrm{m}$ thick sections). The slices were pre-incubated with $0.5 \%$ Triton X-100 and $0.1 \%$ bovine serum albumin, and then dual immunohistochemistry was performed. Briefly, we used rat anti-CldU antibody (Accuratechemicals 1:500) and a mouse anti-IdU antibody (BD Biosciences 1:500) overnight. Primary antibodies were detected by using secondary Alexa-conjugated antibodies from Molecular Probes (1:1,000): Alexa 488 conjugated donkey anti-rat for the anti-CldU antibody, Alexa 594 conjugated donkey anti-mouse for the anti-IdU antibody overnight, and the reverse fluorophoreconjugated antibodies for the control experiments switching the colors of the secondary antibodies. Sections were counterstained finally with DAPI (Calbiochem 1:1,000) for $10 \mathrm{~min}$.

We found and consistently replicate the result that cells were labeled in decreasing numbers with increasing survival time, as expected, and this was consistent for both analogs. In addition, the number of cells dual labeled for CldU and IdU ( $\mathrm{CldU}^{+} / \mathrm{IdU}^{+}$cells) in the same animal also decreases as the time between injections of each thymidine analog increased, as is expected (see Figure 2 taken from Llorens-Martin et al., 2010).
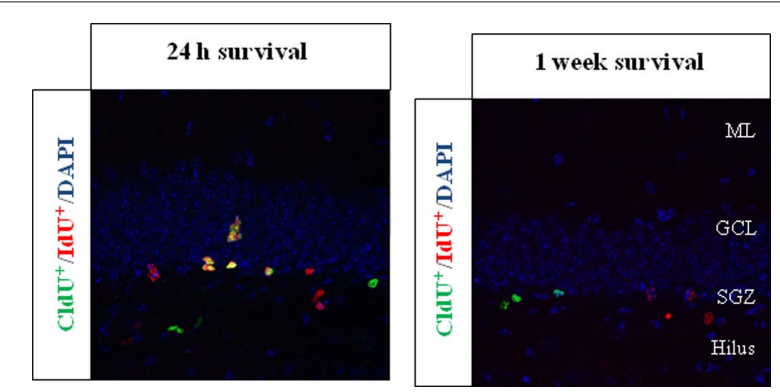

FIGURE 2 | Representative pictures of $\mathrm{CldU}^{+}$and IdU ${ }^{+}$cells in set-up experiments. The thymidine analogs were injected in the same individuals separated by either $24 \mathrm{~h}$ or 1 week; BrdU-equimolecular dosages of CldU and IdU were injected. Animals were then sacrificed $2 \mathrm{~h}$ after the last injection. We found that the injection of different thymidine analogs separated by more than 1 day ( 1 week survival) led to no overlapping of the labeling, while in the $24 \mathrm{~h}$ experiment a huge proportion of cells were co-labeled, as expected. The labeling was assessed in the hippocampal dentate gyrus of adult mice. The pictures were registered from $50 \mu \mathrm{m}$ thick coronal sections, and both are taken from Llorens-Martin et al. (2010). ML, molecular layer; GCL, granule cell layer; SGZ, subgranular zone. 
Other authors had previously demonstrated the feasibility and reliability of both analogs to produce a replicable and consistent labeling of dividing cells. In this way, Vega and Peterson (2005) showed that simultaneous equimolar delivery of both IdU and CldU co-labeled all cells with both markers. Next they demonstrated that the administration of equimolar concentrations of IdU and CldU 1 day apart is able to label three populations of proliferative cells (one of the markers or both together).

In a different experiment, we changed the order of the injections with respect to our design mentioned above; some animals were injected CldU first and later IdU, while other animals were injected in reverse order. The quality of aforementioned labeling unchanged whatever the order in which the analogs were injected. The number of cells obtained after $24 \mathrm{~h}$ survival either with CldU or IdU, and the number of cells obtained after 2 weeks survival by injecting CldU or IdU, were consistently similar.

Bonaguidi et al. (2008) performed a number of interesting control experiments. By labeling with IdU and CldU separated in time by several weeks, the authors could see in the granule cell layer (GCL) of the hippocampal dentate gyrus that older cells were located farther within the GCL than younger cells, consistent with labeling two temporally distinct precursor populations. Besides, dual-labeled cells were found in very low numbers.

Our experiments have all been performed in mice, like many other authors. However, a number of studies have reported similar protocols, and importantly, similar results about the reliability of the technique by using rats. Problems of specificity, cross-reactivity, and feasibility of the technique, don't therefore seem to rely on the species the experiments have been carried out up to date.

We have never encountered cross-reactivity problems of the antibodies in tissue from mice. Cross-reactivity has not been a major problem frequently reported in the literature, except for the early works by Shibui et al. (1989) by using BrdU and IdU, or the work by Aten et al. (1992) by using CldU and IdU detected by means of anti-BrdU antibodies specific for BrdU-CldU or only BrdU. The former work required complicated histological procedures to ensure the specificity of staining, while the second one required the use of a high-salt buffer to differentially remove binding from each substrate. None of these problems are common today with the commercially available antibodies.

\section{TROUBLESHOOTING}

The simplicity of the protocols reviewed here makes this technique affordable and easy to implement. There are no special considerations to take into account in dealing with the development of the technique (except for the commentaries on cross-reactivity above mentioned). However, we will mention a couple of minor issues: the first is the consideration on the order in which the analogs are injected into animals in experimental designs aimed at scoring two subpopulations of qualitatively different age. Although we have seen experimentally that CldU/IdU can be administered in any order obtaining identical results in the type and quality of cell labeling, we first administered in our experiments CldU, and IdU later, because the long-term experiments course with a significant non-negligible increase in body weight of animals, which forces to inject large amounts of the second analog. Due to the considerably greater cost of the current commercially available CldU versus IdU, we inject CldU first, when the animals are smaller, allowing to considerably save product.

The second is relative to the dissolution of the IdU. IdU has a low solubility in water. This should not be a problem if one has in mind a couple of considerations. First, the product should be prepared immediately prior to injection, taking great care to its dissolution (see above). Second, if the experiment includes a large number of animals, the product may begin to precipitate and crystallize after half an hour of its dissolution. In this case, the product must be re-diluted, or, if it is expected to spend more than half an hour to inject all animals, the product can be prepared in two batches.

\section{CONCERNS}

The use of thymidine analogs CldU and IdU might hit a last hurdle: the sensitivity and specificity of these analogs was not enough to carry out reliable studies (Leuner et al., 2009). Of course, if the sensitivity is somewhat lower than that of BrdU, it is not a relevant issue if we are not interested in calculating an estimate of the actual number of cells in the tissue under study. If this were the case, the analysis of the data would suffer with this concern. But for comparative studies of experimental animal groups treated equally, the differential sensitivity with respect to BrdU is not relevant, because it is not here to estimate the actual number of cells in $\mathrm{S}$ phase at the time of injection to survive until sacrificing the animal, but rather to compare the apparent number of these cells between experimental groups. This assertion deserves a deeper discussion: it is not that our estimate (the number of labeled cells, obtained by using similar CldU and IdU), is biased. By contrast, the differential relative sensitivities of CldU and IdU, among themselves and with BrdU, and the greater penetration and specificity of the respective antibodies will simply yield estimates of the number of labeled cells that are not intrinsically comparable, but each marker itself is not biased. In a word, different analogs produce different data accuracy in terms of sensitivity, but we have no prime facie evidence that the resulting estimator is biased. Therefore, it is quite useful for comparison between experimental groups. It is not, however, to compare studies from different authors if some use BrdU and other CldU, for example, or if the object of study is to estimate the actual number of surviving cells that were in $\mathrm{S}$ phase at the time of injection.

\section{SOME RESULTS AND DISCUSSION}

Labeling of cell nucleus with the thymidine analog BrdU has been used to identify proliferating cells for years (see for example Wojtowicz and Kee, 2006). The progression through S phase is considered a hallmark event to birthdate a cell. CldU and IdU can reliably be used in the same way (Vega and Peterson, 2005). "Because neurons are strictly postmitotic, a BrdU-positive neuron that is detected some time after BrdU injection must have originated from a cell that divided at exactly the time when BrdU was systemically available ( sic)" (Kempermann, 2006). CldU and IdU are known from 45 years ago, and its potential for dual labeling has been used in cytometry over the last 30 years (for a recent review, see for example Yokochi and Gilbert, 2007). However, its application for the studies of adult neurogenesis 
has not begun until the middle of the last decade, including the analysis of its ability to be used to identify transplanted cells to the central nervous system (see Burns et al., 2006 for a study of the potential for transfer of thymidine analogs from grafted cells to dividing host cells).

\section{CORRELATIONS BETWEEN CELL POPULATIONS OF DIFFERENT AGE}

One of the biggest advantages of studying two cell populations of qualitatively different age in the same animal is that any hypothetical relationship between such populations can be analyzed directly. For example, one obvious and direct relationship, the possible influence of higher or lower rate of survival of an older cell population on the survival rate of the younger cell population, for instance, can be ruled out by analyzing the correlation between the numbers of cells in each population. The higher or lower number of older newborn cells can be correlated significantly with greater or lesser number of younger newborn cells. So we can discard or not a working hypothesis based on the premise that the quantity of one population could affect the survival of the other subpopulation. So far, this analysis would be performed by evaluating the effect of a given treatment on two experimental groups made of different animals, each group with a unique different labeling, one group to study a population of age $\mathrm{X}$, and another group to study the population of age $2 \mathrm{X}$, for example. The results would take the following form: the effect of treatment is that the survival of the younger population $\mathrm{X}$ is increased (or vice versa), while the survival of the older population $2 \mathrm{X}$ decreases (or vice versa). In this format, no further conclusions could be drawn, but most important, it is always possible to argue that unknown factors may have influenced the difference in treatment effect, what we call a group effect.

By contrast, with two immunohistochemically compatible markers as CldU and IdU, the analysis could be done by evaluating the effect of treatment on only one group of animals, avoiding the group effect. The results would take the following form: the effect of treatment is that the survival of X increases (or decreases), while 2X survival decreases (or increases), and a statistically significant correlation (or not) exists between both populations, in the sense that the animals with a higher number of $2 \mathrm{X}$ old cells, have fewer $\mathrm{X}$ old cells, for example. This conclusion alone allows to assess a hypothesis formulated as "the number of adult-born neurons surviving a treatment or condition in a given brain region, affects or modulates the survival of neurons born at a later stage in the same region."

A quantitative approach as specified, on the number of new neurons, can also be adapted to assess a number of different morphological features, such as the degree of maturation of new neurons, the expression of other markers of differentiation, the number of dendritic branches, the number of synaptic buttons, etc. Such an approach has been recently used by Tronel et al. (2010), to demonstrate that spatial learning influences the development of the dendritic trees of the newborn neurons through different, interrelated ways depending on the age and differentiation stage of the different subpopulations of new cells.

All assumptions made about the possible influence of a population of old new neurons on young new neurons arise from the possibility of studying both subpopulations coexisting together in the same tissue, coexisting in a single section of one animal, in the same way as suspects must be identified in a line-up in which they are all placed together in front of the witness. This method, when possible, is significantly more reliable than attempting to identify suspects by just looking at them one at a time. Similarly, any comparison of different populations is most effective when performed in the same animal. This immediately rules out possible artifacts or unknown interfering factors that could contribute to the effects observed in different animals.

In a recent study, our group addressed the issue that environmental enrichment (EE) specifically modulates hippocampal neurogenic cell populations over time (Llorens-Martin et al., 2010). We used the dual-birthdating to study two subpopulations of newborn neuron in mice: those born at the beginning and at the end of enrichment. In this way, we demonstrate that while short-term cell survival is upregulated after an initial 1 week period of enrichment, after long-term enrichment ( 2 months) neither cell proliferation nor the survival of the younger newly born cell populations are distinguishable from that observed in non-enriched control mice. In addition, we show that the survival of older newborn neurons alone (i.e., those born at the beginning of the enrichment) is higher than in controls, due to the significantly lower levels of cell death. These findings suggested an early selective, long-lasting effect of EE on the neurons born in the initial stages of enrichment. Therefore, we could conclude that EE induces differential effects on distinct subpopulations of newborn neurons depending on the age of the immature cells.

A different and elegant approach of this analysis was used by Bauer and Patterson (2005) to distinguish between nucleoside incorporation due to either cell proliferation or DNA repair/apoptosis, by injection of IdU and CldU before and after brain irradiation, respectively. With this approach, the authors were able to compare the putative incorporation of nucleoside during DNA repair (after irradiation, through a possible increase in CldU labeling due to cell proliferation plus DNA repair) and the IdU incorporation (due to cell proliferation only). They reported evidences that nucleosides are not significantly incorporated during DNA repair in the adult brain, and that labeling is not detected in dying postmitotic neurons.

Similarly, CldU and IdU injection before or after EE or running were used to analyze the promoting effect of physical-cognitive activity on the different cell subpopulations involved in the neurogenic process, by means of a complete battery of neurogenic markers (Gobeske et al., 2009). Thus, CldU or IdU labeling, together with cell lineage identity assessment (by means of Sox 2 or GFAP for NSC, nestin for early progenitors, doublecortin for young neurons, and NeuN for mature neurons) enabled the authors to conclude the role of the signaling pathway they were interested (BMP) in the neurogenic process and its role as a mediator of the procognitive and proneurogenic effects of enrichment and exercise. Importantly, they demonstrated that both exercise and noggin increased proliferation across the lineage: from progenitors to neuroblasts, but also that increased medium-term survival (7-10 days). This work is a very good example of how the dual-birthdating technique can be combined with a battery of molecular markers to better characterize the cellular subpopulations under study. 
Obviously, the approaches listed here are only the first assays using CldU and IdU (together with other techniques) that have been published applied to the analysis of adult neurogenesis. Many others are possible and will be published shortly. Just to mention a few of them, one interesting possibility might be the use of retroviruses to infect dividing cells in the dentate GCL of adult animals. Recently, Tronel et al. (2010) published an interesting study demonstrating that learning-induced changes in new neurons are long-lasting and specific to adult-born neurons in the dentate gyrus. For this purpose, the authors labeled newborn neurons by infecting the dentate gyrus with a retrovirus with a ubiquitous, long-term GFP-expressing promoter. On the same day, animals were administered BrdU. This way the authors could show the influence of learning on the length of dendrites of 3-month old newborn neurons (BrdU labeled). This type of analysis will be extended further in the near future by the use of dual-birthdating, by incorporating both CldU and IdU, and retrovirus infection at different time points. A recent review about these last approaches and its combination with dual-birthdating markers has been published by Landgren and Curtis (2010).

A different approach has been used to distinguish between newborn granule neurons in the adult dentate gyrus (labeled with IdU) and the granule neurons born during development (CldU). With this technique, Stone et al. (2010) have demonstrated that the integration rates of dentate granule neurons into memory networks does not appear to depend on the developmental stage of generation of granule cells, and that a functional equivalence between the different developmental stages exist.

\section{DUAL-BIRTHDATING AND BEHAVIOR}

The comparison of two neuronal populations of different ages in the same animal can be particularly informative when combined with behavioral analyses. Until very recently, and even today, many studies have evaluated the effect of a treatment or an animal's condition on their behavior and on neurogenesis in separate groups of animals. Thus, these studies were required to consider the possible effects of behavioral testing on the survival or proliferation of newborn neurons. Many other studies accounted for this possible influence by quantifying neurogenesis both in groups of animals undergoing behavioral tests and in untested animals (see e.g., Llorens-Martin et al., 2007). It is obvious that after taking into account the possible effect of behavior on neurogenesis, the most interesting studies of neurogenesis and behavior should be carried out in the same animals. For example, it is of great interest to determine whether a treatment or condition has anxiolytic and/or procognitive effects, and increases adult hippocampal neurogenesis in the same animal. However, this whole approach should be made in duplicate to assess a second subpopulation of new neurons of different age. Up to date, the problem was that in this case, it could always be argued that they were different animals, and that the response in behavioral tests could differ between experimental groups in a way that influenced neurogenesis.

In contrast, using two immunohistochemically compatible markers in the same animal, which also undergoes a behavioral testing, it is now possible to test the hypothesis whether the implementation of a behavior, or the effect of a treatment or condition on such behavior is related to a variation in an old or young neurogenic population and not the other, because everything is quantified in the same animal (taken into account once again the possibility of the influence of behavioral tests on neurogenesis, as already mentioned).

One of the earliest and best examples about how to take advantage of this kind of approach is the elegant work by the laboratory of Nora Abrous (Dupret et al., 2007). The authors injected rats with IdU 7 days before a water maze training in order to label the newborn cells for which survival should be increased by learning. CldU was injected 3 days before the start of the training in order to label newly born cells that, as stated in the working hypothesis, might die as a consequence of learning. They found that learning promoted the survival of IdU-labeled cells generated 1 week before exposure to the task, while decreased the survival of CldU-labeled cells that were born 3 days before the start of the training. Both groups of data were associated with the corresponding decreases and increases in positive labeled pyknotic nuclei, respectively. This way the authors showed that learning-induced increases in survival and apoptosis of newborn cells are interrelated processes, and that learning promotes survival of relatively mature neurons, apoptosis of more immature cells, and finally, proliferation of neural precursors.

\section{DIFFERENT STIMULI OR RE-EXPOSITION TO A STIMULUS AND DUAL LABELING OF NEWBORN NEURONS}

A different and interesting target where the present approach can be quite useful deals with the re-exposition of animals to given stimuli. Our laboratory has addressed recently the issue whether the antidepressant and proneurogenic effects of EE are different in animals that are naïve or pre-exposed to the stress inducing helplessness, and whether these differential effects are exerted on distinct neurogenic subpopulations (Llorens-Martín, Tejeda and Trejo, submitted). The repeated exposure to a forced swimming stressor was analyzed together with the effects of EE on different neurogenic populations distinguished by age and differentiation state, by means of using CldU and IdU at separated times in the same animals. We have found that younger cells are more sensitive and responsive to the conditions, both the positive and negative effects. These data will be relevant to identify the cell populations that are the targets of stress, depression, and enrichment, and that form part of the mechanism responsible for mood dysfunctions.

\section{TEMPORAL DISCRIMINATION OF CELL CYCLE}

All the approaches described so far takes advantage of the use of the two markers in distinct neuronal populations generated at distinct times. But a second approach that is now possible to address through the use of two immunohistochemically compatible markers, benefits from the use of the two markers very close in time. A first benefit of such an approach is to analyze the effect of a given treatment on proliferation or immediate survival, compared with the effect on short-term survival. An interesting study that took advantage of this benefit of dual labeling is the work by Thomas et al. (2007). The authors tested the hypothesis whether an acute psychosocial stress affected proliferation, short-term survival or immediate survival. They used sequential thymidine analog administration (CldU and IdU) as follows: three consecutive days of twice-daily (every $12 \mathrm{~h}$ ) injections of either IdU (the first 2 days) 
or CldU (the third day). Next, the animals were sacrificed either on day forth or 7 days later. This way, the authors were able to temporally discriminate DNA replication, by administering equimolar doses of CldU and IdU that, in turn, were equimolar of BrdU usual doses. As stress session took place in the third day, when CldU was injected, the authors could demonstrate that short-term survival but not initial proliferation or immediate survival was altered in response to stress.

\section{RE-ENTERING THE CELL CYCLE}

The use of two deoxyuridines has been used to analyze cell populations able to eventually re-enter the cell cycle. Adult neurogenic niches are characterized for having a rapidly dividing population of proliferative progenitors, and a slowly dividing population of NSC that maintain themselves and generate the progenitors; the latter population therefore retain for longer times a nucleoside label. The approach consists of an initial period of administration of the first analog (2-3 weeks) during which NSC, proliferative progenitors, and a proportion of more differentiated cells will become labeled. However, only some cells will retain labeling, either slowly dividing NSC or differentiated cells (cells which did not dilute the labeling due to active division). The surviving population that retain label for long periods can next be distinguished by administration of a 2-3 weeks second round of a second analog, that will be incorporated only by NSC. Maslov et al. (2004) used this approach with IdU and CldU to establish whether cells that retain a first nucleoside over long periods eventually re-enter the cell cycle in the subependymal zone (SVZ) of the lateral ventricles. Bonaguidi et al. (2008) did the same in the SGZ of the hippocampal dentate gyrus, to demonstrate that the SGZ contains a small population of slowly dividing NSC.

With a similar approach, other works have analyzed the distinct subpopulations of newborn cells able to exit or re-enter the cell cycle after a manipulation of a putative regulative factor is made [like for example in the study of Notch by Breunig et al. (2007)]. And recently, the different properties, morphologies, and mechanisms controlling maintenance and differentiation of distinct subpopulations of NSC in the adult dentate gyrus GCL, has been explored by Lugert et al. (2010) by using CldU and IdU separated by 1-6 or 11 days in different sets of experiments, to demonstrate that two distinguishable populations of NSC exist in the SGZ (quiescent and actively dividing), and importantly, some of these cells can reversibly change its properties to reconstitute the quiescent or the dividing subpopulations, a process involving Notch.

By using the same reasoning above mentioned, this approach let Mira et al. (2010) demonstrate the delicate equilibrium between neural stem cell proliferation and quiescence in the SGZ, supposedly intended to prevent the premature depletion of NSC activity in the mature brain. The authors used the BMP signaling pathway and noggin in a way similar to that above mentioned, to study the consequences of depleting the pool of quiescent NSC. They used CldU at the time when noggin was administered, and IdU several weeks later, thereby $\mathrm{CldU}^{+}$cells retaining label are either NSCs that do not dilute the nucleoside or cells that have matured to granule neurons. That way, the authors could analyze the NSCs that re-entered the cell cycle by means of the dual labeling with IdU. Some other authors have used the same strategy, for example to analyze the effect of LIF on NSCs from ventricular zone (Bauer and Patterson, 2006). A recent review of these approaches and its technical details has been published (Tuttle et al., 2010).

A second benefit stems from the fact that whether the time difference in the administration of the two markers is short enough, it is likely that a proportion of cells that captured the first analog during $S$ phase of cell cycle can be detected in a new round of $S$ phase of the cycle with the second analog. This possibility opens the door to studies in which a particular treatment or condition may have an influence on cell cycle re-entry in neurogenic subpopulations during short time periods, and to study the intermediate progenitor cells subpopulation. One such study has been published recently (Brandt et al., 2010), designed to distinguish between cell cycle exit and continued division at the progenitor cell level. Specifically, the authors analyze the proportion of type- $2 \mathrm{~b} / 3$ cells re-entering $S$ phase by labeling the cells by means of the two thymidine analogs. This way, Brandt and collaborators have demonstrated that running promotes both proliferation and cell cycle exit of $\mathrm{DCX}^{+}$ type-3 precursors.

\section{MULTIPLE LABELING}

Recent work (Chehrehasa et al., 2009) has shown the possibility to label two different populations of dividing cells by means of BrdU (revealed by immunohistochemistry as mentioned), and EdU (the thymidine analog 5-ethyl-2'-deoxyuridine, detected by click chemistry; Bradford and Clarke, 2011). However, several laboratories including ours are experiencing some complications to implement triple labeling including EdU, because some of the commercially available antibodies against CldU or IdU cross-react with EdU.

We do not doubt that soon we will see the solution of these transient technical problems and we will witness the chance to label more than two subpopulations of cycling cells in the same animal by using three markers. The only limits will be the number of both secondary antibodies and colors separated enough to be clearly differentiated in the confocal microscope we can get.

\section{CONCLUSION}

We think that the possibility of reliably detecting dual labels of cell birthdating ("birth-marking" labels; Kempermann, 2006) represents a qualitative improvement in the tools available to analyze adult neurogenesis, as we demonstrate in the studies cited here. The ability to unequivocally identify separate subpopulations of newborn neurons, according to their age, each with putative different maturational states and in turn, different properties, will continue to improve (however, see the interesting insight by Eisch and Mandyam, 2007) advancing in the analysis of the role/s of the immature new neurons, because the properties of different populations of newborn neurons and their influence in the performance of different behaviors will be fully understood only when studied in the same animal.

\section{ACKNOWLEDGMENT}

The authors wish to thank Gonzalo S. Tejeda for his help with the setup and performance of some experiments mentioned in this manuscript, Dr. Mark Sefton for his assistance in revising the manuscript, and Simona Gradari for her contribution to the drawings in Figure 1. This work was supported by a grant from Spanish Ministerio de Educación y Ciencia, BFU2007-60195/BFI, to José L. Trejo. 


\section{REFERENCES}

Aimone, J. B., Deng, W., and Gage, F. H. (2010). Adult neurogenesis: integrating theories and separating functions. Trends Cogn. Sci. (Regul. Ed.) 14, 325-337.

Aten, J.A., Bakker, P. J., Stap, J., Boschman, G.A., and Veenhof, C. H. (1992). DNA dual labelling with IdUrd and CldUrd for spatial and temporal analysis of cell proliferation and DNA replication. Histochem. J. 24, 251-259.

Bakker, P. J., Stap, J., Tukker, C. J., van Oven, C. H., Veenhof, C. H., and Aten, J. (1991). An indirect immunofluorescence dual staining procedure for the simultaneous flow cytometric measurement of iodo- and chlorodeoxyuridine incorporated into DNA. Cytometry 12, 366-372.

Bauer, S., and Patterson, P. H. (2005). The cell cycle-apoptosis connection revisited in the adult brain. J. Cell Biol. 171, 641-650.

Bauer, S., and Patterson, P. H. (2006). Leukemia inhibitory factor promotes neural stem cell self-renewal in the adult brain. J. Neurosci. 26, 12089-12099.

Bonaguidi, M.A., Peng, C.Y., McGuire, T., Falciglia, G., Gobeske, K. T., Czeisler, C., and Kessler, J. A. (2008). Noggin expands neural stem cells in the adult hippocampus. J. Neurosci. 28, 9194-9204.

Bradford, J. A., and Clarke, S. T. (2011). Dual-pulse labeling using 5-ethynyl2 -deoxyuridine (EdU) and 5-bromo2 -deoxyuridine (BrdU) in flow cytometry. Curr. Protoc. Cytom. Chapter 7, Unit7 38, PMID 21207361.

Brandt, M. D., Maass, A., Kempermann, G., and Storch, A. (2010). Physical exercise increases Notch activity, proliferation and cell cycle exit of type-3 progenitor cells in adult hippocampal neurogenesis. Eur. J. Neurosci. 32, 1256-1264.

Breunig, J. J., Silbereis, J., Vaccarino, F. M., Sestan, N., and Rakic, P. (2007). Notch regulates cell fate and dendrite morphology of newborn neurons in the postnatal dentate gyrus. Proc. Natl. Acad. Sci. U.S.A. 104, 20558-20563.

Burns, K. A., and Kuan, C. Y. (2005). Low doses of bromo- and iododeoxyuridine produce near-saturation labeling of adult proliferative populations in the dentate gyrus. Eur. J. Neurosci. 21, 803-807.
Burns, T. C., Ortiz-Gonzalez, X. R., Gutierrez-Perez, M., Keene, C. D. Sharda, R., Demorest, Z. L., Jiang, Y., Nelson-Holte, M., Soriano, M. Nakagawa, Y., Luquin, M. R., GarciaVerdugo, J. M., Prosper, F., Low, W. C., and Verfaillie, C. M. (2006). Thymidine analogues are transferred from prelabeled donor to host cells in the central nervous system after transplantation: a word of caution. Stem Cells 24, 1121-1127.

Chehrehasa, F., Meedeniya, A. C., Dwyer P., Abrahamsen, G., and Mackay-Sim, A. (2009). EdU, a new thymidine analogue for labelling proliferating cells in the nervous system. J. Neurosci. Methods 177, 122-130.

Deng, W., Aimone, J. B., and Gage, F. H (2010). New neurons and new memories: how does adult hippocampal neurogenesis affect learning and memory? Nat. Rev. Neurosci. 11,339-350.

Dupret, D., Fabre, A., Dobrossy, M. D., Panatier, A., Rodriguez, J. J., Lamarque, S., Lemaire, V., Oliet, S. H., Piazza, P. V., and Abrous, D. N. (2007). Spatial learning depends on both the addition and removal of new hippocampal neurons. PLoS Biol. 5, e214. doi: 10.1371/ journal.pbio.0050214

Eisch, A. J., and Mandyam, C. D. (2007) Adult neurogenesis: can analysis of cell cycle proteins move us "Beyond BrdU"? Curr. Pharm. Biotechnol. 8, 147-165.

Gobeske, K. T., Das, S., Bonaguidi, M. A., Weiss, C., Radulovic, J., Disterhoft, J. F., and Kessler, J. A. (2009). BMP signaling mediates effects of exercise on hippocampal neurogenesis and cognition in mice. PLoS One 4, e7506. doi: 10.1371/journal.pone.0007506

Hoshino, T., Ito, S., Asai, A., Shibuya, M. Prados, M. D., Dodson, B. A., Davis, R. L., and Wilson, C. B. (1992). Cell kinetic analysis of human brain tumors by in situ dual labelling with bromodeoxyuridine and iododeoxyuridine. Int. J. Cancer 50, 1-5.

Kempermann, G. (2006). Adult Neurogenesis. New York: Oxford University Press.

Landgren, H., and Curtis, M. A. (2010) Locating and labeling neural stem cells in the brain. J. Cell. Physiol. 226, 1-7.

Leuner, B., Glasper, E. R., and Gould, E. (2009). Thymidine analogue methods for studies of adult neurogenesis are not equally sensitive. J. Comp. Neurol. 517, 123-133.
Llorens-Martin, M., Tejeda, G. S., and Trejo, J. L. (2010). Differential regulation of the variations induced by environmental richness in adult neurogenesis as a function of time: a dual birthdating analysis. PLoS One 5, e12188. doi: 10.1371/journal. pone. 0012188

Llorens-Martin,M.V., Rueda, N., MartinezCue, C., Torres-Aleman, I., Florez, J. and Trejo, J. L. (2007). Both increases in immature dentate neuron number and decreases of immobility time in the forced swim test occurred in parallel after environmental enrichment of mice. Neuroscience 147, 631-638.

Lugert, S., Basak, O., Knuckles, P., Haussler, U., Fabel, K., Gotz, M., Haas, C.A., Kempermann, G., Taylor, V., and Giachino, C. (2010). Quiescent and active hippocampal neural stem cells with distinct morphologies respond selectively to physiological and pathological stimuli and aging. Cell Stem Cell 6, 445-456.

Manders, E. M., Stap, J., Brakenhoff, G. J. van Driel, R., and Aten, J. A. (1992). Dynamics of three-dimensional replication patterns during the S-phase, analysed by dual labelling of DNA and confocal microscopy. J. Cell. Sci. 103 857-862.

Maslov, A. Y., Barone, T. A., Plunkett, R. J., and Pruitt, S. C. (2004). Neural stem cell detection, characterization, and age-related changes in the subventricular zone of mice. J. Neurosci. 24 1726-1733.

Mira, H., Andreu, Z., Suh, H., Lie, D C., Jessberger, S., Consiglio, A., San Emeterio, J., Hortiguela, R., MarquesTorrejon, M.A., Nakashima, K., Colak, D., Gotz, M., Farinas, I., and Gage, F. H (2010). Signaling through BMPR-IA regulates quiescence and long-term activity of neural stem cells in the adult hippocampus. Cell Stem Cell 7, 78-89. Shibui, S., Hoshino, T., Vanderlaan, M. and Gray, J. W. (1989). Dual labeling with iodo- and bromodeoxyuridine for cell kinetics studies. J. Histochem. Cytochem. 37, 1007-1011.

Stone, S. S., Teixeira, C. M., Zaslavsky, K. Wheeler, A. L., Martinez-Canabal, A. Wang, A. H., Sakaguchi, M., Lozano, A. M., and Frankland, P. W. (2010). Functional convergence of developmentally and adult-generated granule cells in dentate gyrus circuits supporting hippocampus-dependent memory. Hippocampus, doi: 10.1002/ hipo.20845. [Epub ahead of print].

Thomas, R. M., Hotsenpiller, G., and Peterson, D. A. (2007). Acute psychosocial stress reduces cell survival in adult hippocampal neurogenesis without altering proliferation. J. Neurosci. 27, 2734-2743.

Tronel, S., Fabre, A., Charrier, V., Oliet, S. H., Gage, F. H., and Abrous, D. N. (2010). Spatial learning sculpts the dendritic arbor of adult-born hippocampal neurons. Proc. Natl. Acad. Sci. U.S.A. 107, 7963-7968.

Tuttle, A. H., Rankin, M. M., Teta, M., Sartori, D. J., Stein, G. M., Kim, G. J., Virgilio, C., Granger,A.,Zhou, D., Long, S. H., Schiffman, A. B., and Kushner, J. A. (2010). Immunofluorescent detection of two thymidine analogueues (CldU and IdU) in primary tissue. $J$. Vis. Exp. 46, 2166.

Vega, C. J., and Peterson, D. A. (2005). Stem cell proliferative history in tissue revealed by temporal halogenated thymidine analogue discrimination. Nat. Methods 2, 167-169.

Wojtowicz, J.M., and Kee, N. (2006). BrdU assay for neurogenesis in rodents. Nat Protoc. 1, 1399-1405.

Yokochi, T., and Gilbert, D. M. (2007). Replication labeling with halogenated thymidine analogues. Curr. Protoc. Cell Biol. Chapter 22, Unit 22 10, PMID 18228503

Conflict of Interest Statement: The authors declare that the research was conducted in the absence of any commercial or financial relationships that could be construed as a potential conflict of interest.

Received: 20 January 2011; accepted: 17 May 2011; published online: 27 May 2011. Citation: Llorens-Martín $M$ and Trejo $J L$ (2011) Multiple birthdating analyses in adult neurogenesis: a line-up of the usual suspects. Front. Neurosci. 5:76. doi: 10.3389/fnins.2011.00076

This article was submitted to Frontiers in Neurogenesis, a specialty of Frontiers in Neuroscience.

Copyright (c) 2011 Llorens-Martín and Trejo. This is an open-access article subject to a non-exclusive license between the authors and Frontiers Media SA, which permits use, distribution and reproduction in other forums, provided the original author and source are credited and other Frontiers conditions are complied with. 